\title{
Fluid-based assays and precision medicine of cardiovascular diseases: the 'hope' for Pandora's box?
}

\author{
Giuditta Benincasa (1) , ${ }^{1}$ Gelsomina Mansueto (1) , ${ }^{2}$ Claudio Napoli ${ }^{1,3}$
}

- Additional material is published online only. To view, please visit the journal online (http://dx.doi.org/10.1136/ jclinpath-2019-206178)

${ }^{1}$ Clinical Department of Internal Medicine and Specialistics, Department of Advanced Clinical and Surgical Sciences, University of Campania "Luigi Vanvitelli", Naples, Italy 2Pathology Section, Department of Advanced Biomedical Sciences, University of Naples "Federico II", Naples, Italy ${ }^{3}$ IRCCS-SDN, Naples, Italy

\section{Correspondence to}

Dr Giuditta Benincasa, Naples 80132, Italy; dr.benincasa. giuditta@gmail.com

Received 21 August 2019 Revised 19 September 2019 Accepted 20 September 2019 Published Online First 14 October 2019
ABSTRACT

Progresses in liquid-based assays may provide novel useful non-invasive indicators of cardiovascular (CV) diseases. By analysing circulating cells or their products in blood, saliva and urine samples, we can investigate molecular changes present at specific time points in each patient allowing sequential monitoring of disease evolution. For example, an increased number of circulating endothelial cells may be a diagnostic biomarker for diabetic nephropathy and heart failure with preserved ejection fraction. The assessment of circulating cell-free DNA (cfDNA) levels may be useful to predict severity of acute myocardial infarction, as well as diagnose heart graft rejection. Remarkably, circulating epigenetic biomarkers, including DNA methylation, histone modifications and non-coding RNAs are key pathogenic determinants of CV diseases representing putative useful biomarkers and drug targets. For example, the unmethylated FAM101A gene may specifically trace cfDNA derived from cardiomyocyte death providing a powerful diagnostic biomarker of apoptosis during ischaemia. Moreover, changes in plasma levels of circulating miR-92 may predict acute coronary syndrome onset in patients with diabetes. Now, network medicine provides a framework to analyse a huge amount of big data by describing a CV disease as a result of a chain of molecular perturbations rather than a single defect (reductionism). We outline advantages and challenges of liquid biopsy with respect to traditional tissue biopsy and summarise the main completed and ongoing clinical trials in CV diseases. Furthermore, we discuss the importance of combining fluid-based assays, big data and network medicine to improve precision medicine and personalised therapy in this field.

\section{INTRODUCTION}

It is, in fact, nothing short of a miracle that the modern methods of instruction have not yet entirely strangled the holy curiosity of inquiry; for this delicate little plant, aside from stimulation, stands mainly in need of freedom. Without this it goes to wrack and ruin without fail. - Albert Einstein

According to the myth, curiosity led Pandora to open the forbidden box without knowledge of the consequences and when she closed it only the spirit of hope was left inside. Likewise, the innate curiosity of generations of researchers is advancing the current paradigms in the cardiovascular (CV) clinical setting and the major 'hope' is that scientific discovery drives clinical delivery. The database DisGeNet (http://www.disgenet.org) ${ }^{1}$ is a useful platform that currently lists over 1000 candidate genes involved in the aetiology of several CV diseases; however, for most of them the underlying molecular mechanisms remain to be clarified and more effective and more personalised therapies are needed. ${ }^{2-5}$ The tremendous progress in different omics platforms, including next-generation sequencing (NGS), epigenomics, proteomics, metabolomics and foodome mapping has allowed a molecular quantification of genotype-environment relationship and nutritional habits for $\mathrm{CV}$ diseases at an unprecedented level of resolution. ${ }^{6-9}$ These efforts have generated a huge amount of big data and consequent computational challenges. In spite of these difficulties, potent bioinformatics algorithms are able to better interpret and integrate this large volume of data. Indeed, these are offering multilevel network analyses to identify key pathogenic nodes, including genes, protein, metabolites and their interactions in the human interactome, useful as innovative biomarkers and drug targets. ${ }^{10-13}$ Network medicine is an integrative molecularbioinformatic approach useful to advance the current medical practices by overcoming the limitations of the reductionist concept viewing a disease as a direct consequence of a single molecular defect, still central in CV care. ${ }^{71-14}$ Moreover, machine learning techniques applied to complex electronic health records are being used in clinical trials (NCT00303212) to develop prediction models useful to diagnosis, classification, readmissions and medication adherence in patients with heart failure (HF). ${ }^{15}$ In order to translate mechanistic insights into everyday CV clinical practice, human specimens should be collected in biobanks during large phase II-III trials, which may confirm or not the expected impact of molecular strategies on longterm outcomes. ${ }^{16-18}$ Liquid biopsy is defined as an easy sampling of different human fluids, mainly blood, saliva and urine, to analyse a large spectrum of putative circulating non-invasive biomarkers. However, this definition is a limitation in the existing literature because the term 'biopsy' usually refers to isolation of tissues. Rather, advanced fluidbased assays better describe the modern complex technologies able to detect circulating intact cells and cell-free nucleic acids, circulating epigeneticsensitive molecules (DNA methylated, modified histones and non-coding RNAs), circulating metabolites and other cell products, such as microvesicles and exosomes in CV diseases. ${ }^{19-22}$ Could liquid biopsy represent the 'the hope' for Pandora's box in precision medicine of $\mathrm{CV}$ diseases? We summarise the main advantages and limitations of several liquid biopsy approaches with respect to traditional endomyocardial biopsy (EMB) and discuss their putative clinical applications for personalised therapy of 
some CV diseases, mainly coronary heart disease (CHD), acute myocardial infarction (AMI), HF and pulmonary arterial hypertension (PAH). Moreover, we discuss that integrated epigenetic/ imaging approaches and network medicine might enhance this field.

\section{A FOCUS ON ENDOMYOCARDIAL BIOPSY DIAGNOSTICS}

Traditional EMB, established in parallel with the development of percutaneous catheter technology in 1960, is often used as the gold standard invasive technique for the differential diagnosis of many primary and secondary CV diseases including cardiomyopathies, myocarditis, infiltrative lesions, arrhythmias and drug toxicities, as well as to monitor allograft rejection after heart transplantation (table 1). ${ }^{2324}$ The tissue sample procured is generally a $1-4 \mathrm{~mm}$ of endocardium and myocardium, which allows the examination of multiple serial sections in order to evaluate endocardium, myocardium, interstitium and vasculature architecture $^{23-25}$ (figure 1). EMB with vascular access is an invasive highly specialised procedure that requires competence and has potential variability in execution. Typically, EMB is performed through the jugular or, more rarely, femoral veins and is clearly associated with a low risk of procedural complications. ${ }^{23-25}$ The main limits for a correct histological diagnosis are: 1) the sampling site; indeed, the site of the sampling is generally the septum to prevent complications of wall rupture, not allowing the evaluation of the whole heart, 2) the sensitivity; indeed, EMB is only valuable when it has a positive diagnosis, 3) very small tissue specimens and 4) the variety of heart diseases. ${ }^{23-25}$ EMB is characterised by a good diagnostic accuracy for storage diseases (eg, amyloidosis, haemochromatosis and other storage diseases have a diffuse macroscopic and microscopic model both in systemic and localised forms with few and precise histological features such as amyloid or iron deposition), as they are widespread in all cardiac walls and the diagnosis is not influenced by the sampling site. ${ }^{23-25}$ In contrast, diagnostic accuracy is low in diseases that have a different or favourite localisation or that have different morphological aspects that are all necessary to evaluate for a complete histological diagnosis (eg, AMI, arrhythmogenic right ventricular dysplasia (ARVD)). ${ }^{23-25}$ In fact, in ARVD, the right ventricle is more affected then the left one, but the involvement of the latter is reported in several studies; it is characterised by a progressive substitution of myocardiocytes with adipose and/or fibrous tissue and the value for histological diagnosis provides a minimum of $3 \%$ of adipose tissue and $>40 \%$ of fibrous tissue, but many authors believe that a percentage of adipose tissue ranging from $5 \%$ to $20 \%$ is suspicious. ${ }^{26}$ About that, the histological criteria (Task Force Criteria 1994), modified in 2010 provide for microscopic observation at a high magnification $(40 \times)$, of seven fields in five areas of the myocardium with the support of histochemical stains for the fibrous connective tissue and for the adipose tissue. The histological features of seven fields in five areas of the myocardium constitute one of the diagnostic criteria for a correct diagnosis. ${ }^{26}$

In addition, in ARVD the remnant myocardium may appear hypertrophic, and atrophic and the cardiomyocytes may look vacuolated or show coagulation necrosis as the first sign of ischaemic distress and sometimes, lymphoid infiltrates characterised by $\mathrm{CD}^{+}$or $\mathrm{CD} 4^{+}$T-cellular elements may be observed in the periphery. ${ }^{27} 28$ The important feature is the presence of adipose tissue up to the subendocardial part of the ventricular wall. We understand the difficulty of a correct diagnosis on biopsy, especially considering the left and biventricular variants identified and described in the literature, and the morphological expression of hypertrophy, necrosis and lymphocytic infiltrate in differential diagnosis respectively with hypertrophic cardiomyopathy, ischaemic infarction and myocarditis. ${ }^{27} 28$ Therefore, we currently tend to indicate ARVD, the arrhythmogenic genetic cardiac pathology of both ventricles and calling it arrhythmogenic cardiomyopathy. Although there are clear and documented limits, myocardial biopsy remains the method used today in the follow-up of patients with definite myocarditis and in the follow-up of transplanted patients. Regarding myocarditis, the histological diagnosis includes different forms, classified according to the type of inflammatory cell infiltrate: lymphocytic, eosinophilic, polymorphic, giant cell myocarditis and cardiac sarcoidosis. ${ }^{29}$ It is important to emphasise that the first diagnosis in these patients is difficult and in the follow-up there are objective difficulties related to the sampling site and to the heterogeneity of the localisation of the possible new disease. The number of sampled tissue fragments has a significant impact on diagnostic accuracy, especially in the heterogeneous disease. A more accurate diagnosis was described when $\geq 5$ fragments were sampled, and this is complicated. ${ }^{23-25}$ For these reasons, a specific comparison to echo and cardiac magnetic resonance (CMR) should be made during long-term follow-up to assess cardiac function and myocardial tissue. Some authors emphasised that CMR may be useful as screening test before routine EMB, owing to its high sensitivity for clinically diagnosed heart transplant rejection, and could be helpful in cases of negative rejection on the biopsy specimen. ${ }^{30}$ Moreover, two pilot prospective studies suggested some echocardiographic indices for the detection or exclusion of allograft rejection; however, other investigations are needed to determine if the echo-based scores could be used as an adjunct to the myocardial biopsy. ${ }^{31}{ }^{32}$ In addition, molecular analyses can be applied to histological examination of paraffin sections, for example, the most common cardiotropic viruses, such as enteroviruses, adenoviruses, erythrovirus, human herpes virus 6, Epstein-Barr virus and in the Far East also hepatitis $\mathrm{C}$ are determined, quantified and sequenced by using PCR-based methods. ${ }^{33}$ However, the use of PCR to diagnose viruses is extremely limited in both sensitivity and specificity. The success of the molecular investigation is influenced by the location of the disease, the clinical status of the patient, the sampling, the conservation and processing of biopsy and the technical procedure and limits as indicated by the most recent guidelines. ${ }^{29} \mathrm{In}$ situ hybridisation, the gold standard for active viral replication, is limited by having too few probes to cover all existing viruses. Consensus sequence tools do not capture all strains of enteroviruses (eg, EV68, etc). Until NGS panels are robust, the use of molecular mechanism to diagnose viral myocarditis is flawed, and falsely reassures clinicians of the potential autoimmune, hypersensitivity or postviral aspects of myocarditis. ${ }^{34}$

\section{WHICH ADVANTAGES COULD FLUID-BASED ASSAYS OFFER ON EMB?}

Despite EMB representing the traditional gold standard of many CV diagnosis, it only mirrors a single point in time of the disease state. Thus, such a sampling method is inadequate for the comprehensive characterisation of the damaged heart. Moreover, surgical bioptic procedure is hampered by limited repeatability, costs, time as well as patient age or comorbidity. ${ }^{23-25}$ Despite the fact that cardiac tissue biopsies are not easily available from living patients, human tissues may be obtained also from cadavers, suggesting possible development of Human Tissue BioBanks from body donation programme, such as the ongoing organisation at University of Padua (Italy). ${ }^{35}$ Remarkably, tissue samples 


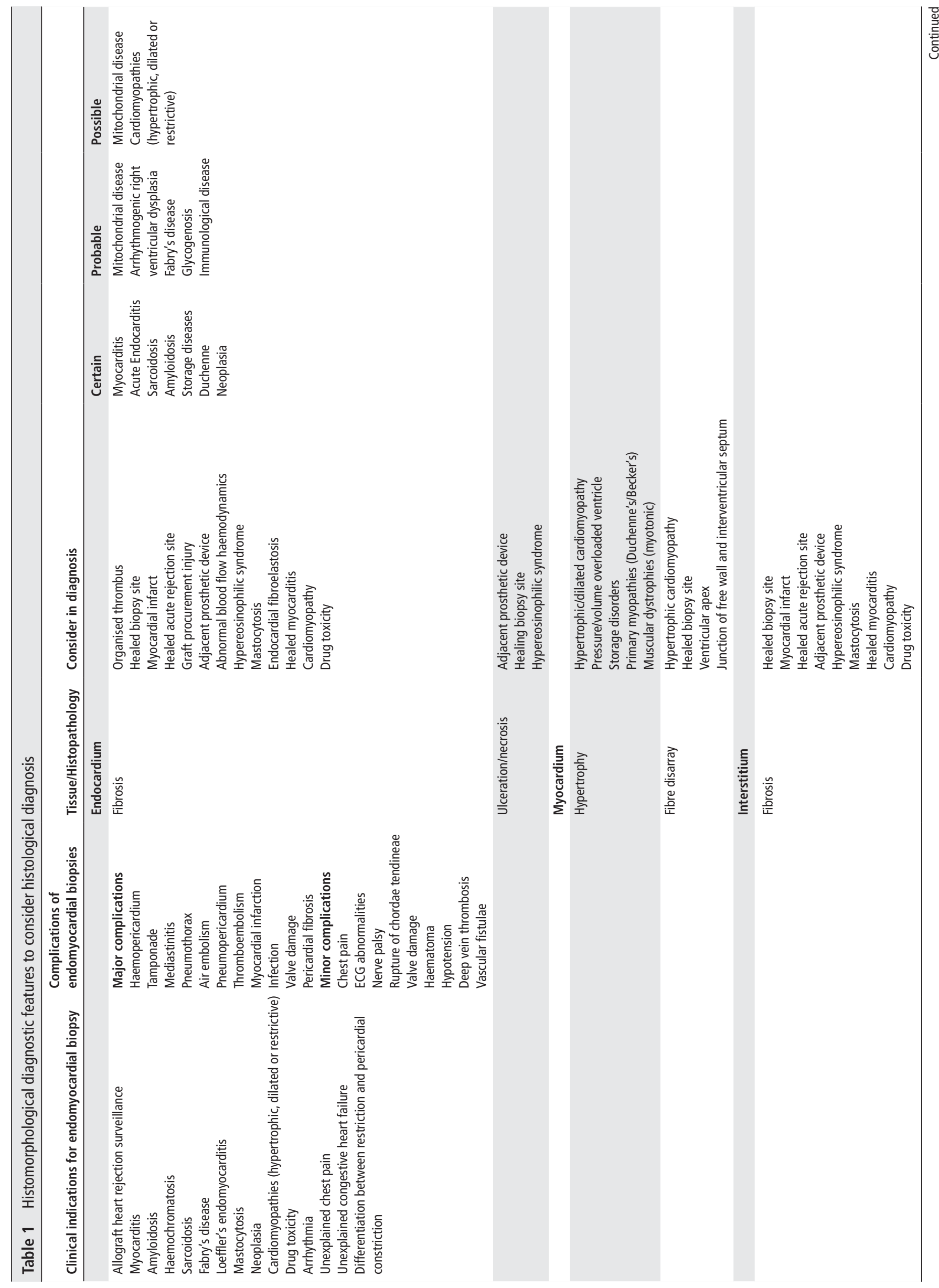




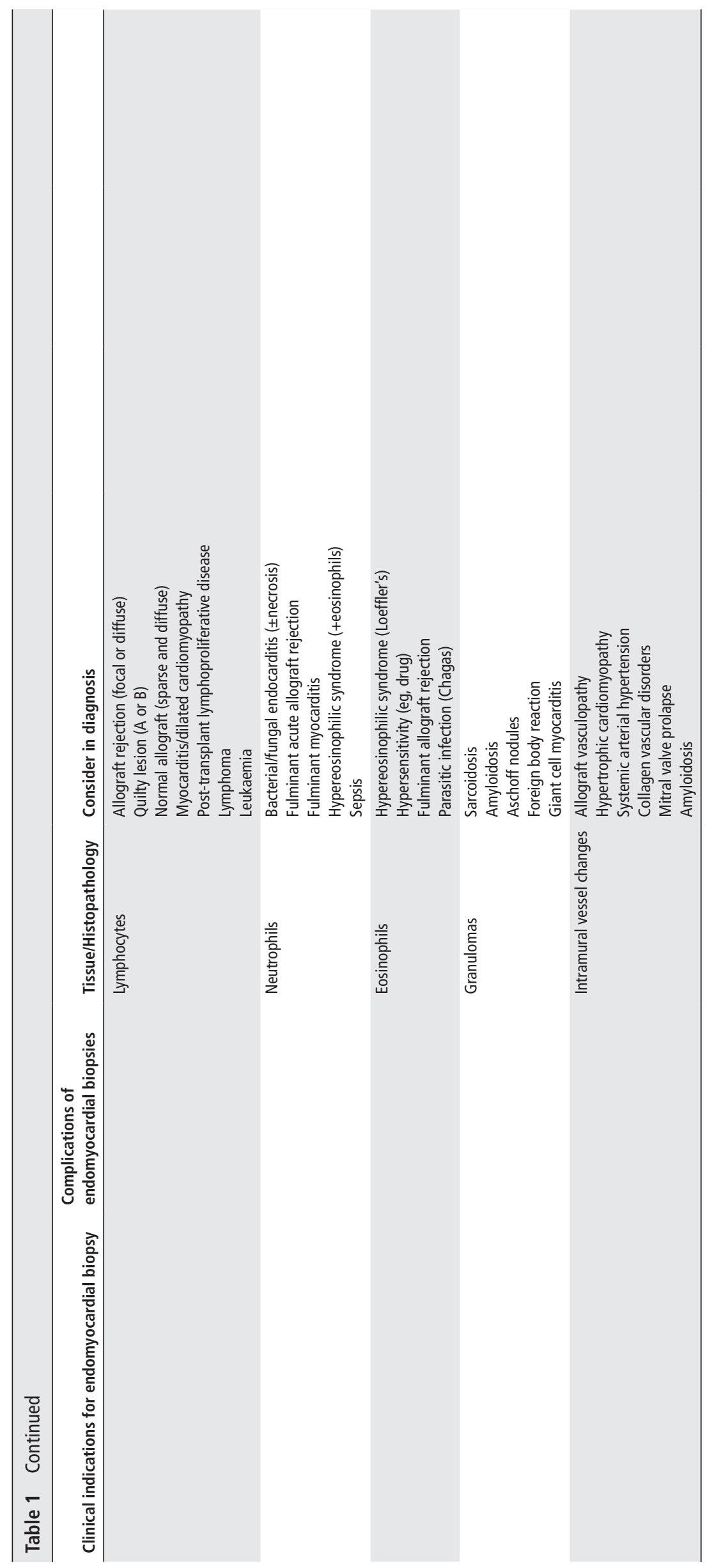

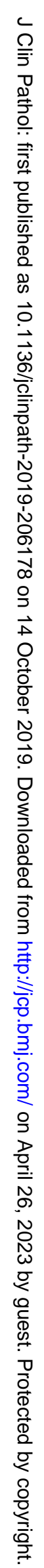




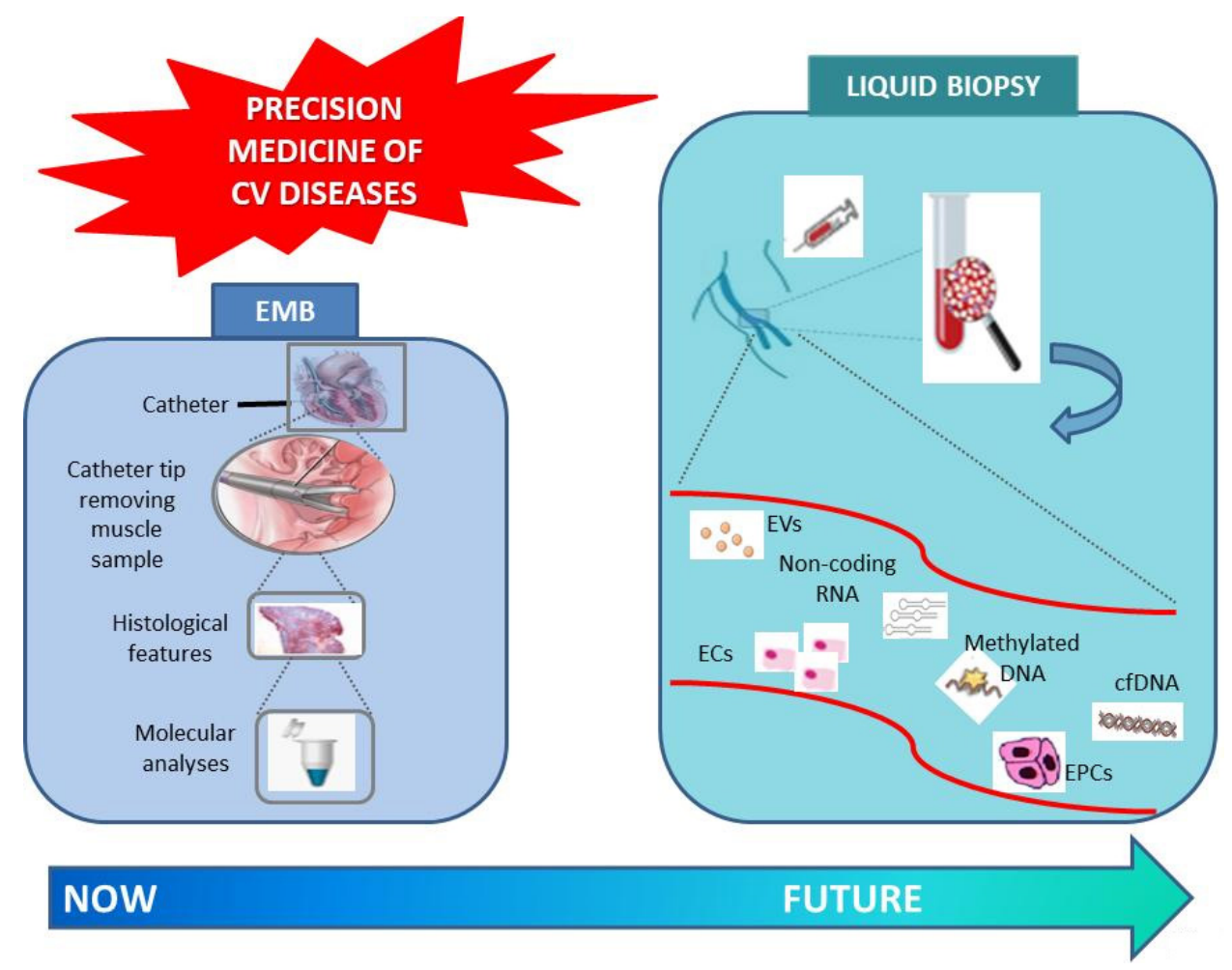

Figure 1 Comparing EMB vs liquid biopsy in CV diseases. On the right panel, we illustrate the procedure of EMB and the downstream histological/ molecular analyses to obtain an accurate diagnosis. On the left panel, we provide a panel of putative clinically informative molecules that can be analysed by blood liquid biopsy. In detail, CECs, EPCs, cell-free nucleic acids (mainly DNA, miRNAs, circRNAs), microvesicles and exosomes derived from a large spectrum of vascular cells could be useful indicators to reach precision medicine and personalised therapy of CV diseases. CECs, circulating endothelial cells; circRNAs, circular RNA; cfDNA, circulating cell-free DNA; CV, cardiovascular; EMB, endomyocardial biopsy; EPCs, endothelial progenitor cells; miRNAs micro-RNAs.

are characterised by a high cell heterogeneity (multiple cell type in tissues) both in physiological and pathological conditions, providing some issues in dissecting the contribution of singular cell types in pathogenesis of CV diseases. In principle, liquid biopsy can dissect the high tissue heterogeneity by informing on many circulating cell types and their products at a specific time point, thus allowing a real-time monitoring of disease evolution (figure 1). Innovative NGS platforms performed on body fluid samples, mainly blood, are providing novel circulating non-invasive biomarkers, which may realise the goals of $\mathrm{CV}$ precision medicine and personalised therapy (figure 1). ${ }^{36}$ These advanced fluid-based strategies offer several advantages: minimally invasive, more quick and easy to obtain, real-time tissue profile, spatiotemporal information and minimal pain or risk. ${ }^{36}$ For these reasons, high complexity liquid-based analytics symbolise a rich source of circulating non-invasive biomarkers to early detect $\mathrm{CV}$ defects before clinical signs occur or before sophisticated imaging systems are able to detect them. In table 2, we summarise advantages and limitations both of liquid biopsy and $\mathrm{EMB}$ in $\mathrm{CV}$ diseases.

\section{PUTATIVE FLUID-BASED APPLICATIONS IN PERSONALISED THERAPY OF CV DISEASES}

Personalised therapy takes into account individual genetic background, environment and lifestyle to more accurately predict which CV treatments and prevention strategies will work better in specific subgroups of patients rather than others. ${ }^{2}$ This collides with a 'one-size-fits-all' approach, in which CV disease treatment and prevention strategies are developed for the average person, with less consideration for the differences among patients. ${ }^{2} 9$ Personalised therapy combines different 'omics' technologies, such as genomics, epigenomics, proteomics and metabolomics as platforms for diagnostic tests, drug discovery and development. ${ }^{279133738}$ Moreover, nanobiotechnology platforms also play an important role in the development of CV personalised therapy enabling diagnosis and treatment with a targeted delivery of therapeutic. ${ }^{39}$ Identification of accurate prognostic and predictive molecular biomarkers discriminating different subgroups of CV patients can improve clinical decisionmaking based on individual molecular profiles. This approach may contribute to enhanced therapeutic efficacy while reducing treatment-related toxicity.

In the last years, great efforts have been put into developing new techniques for fluid-based assays applications as putative useful preventive, diagnostic and prognostic tests in different CV diseases (online supplementary table 1). In figure 2, we summarise the most recent fluid-based applications in $\mathrm{CV}$ diseases, with a major focus on human blood. Moreover, we report some examples of current saliva and urine-based liquid biopsy applications in CV diseases.

\section{Circulating endothelial cells and endothelial progenitor cells}

The circulating form of endothelial progenitor cells (EPCs) originate from bone marrow (BM)-derived haematopoietic stem cells (HSCs) and their reduced number/mobilisation was shown to be linked to CHD. ${ }^{40}{ }^{41}$ Moreover, circulating endothelial cells (CECs) are vascular endothelial cells detected in the peripheral blood of the body under physiological and pathological conditions with a putative role in therapeutic strategies for patients with congenital heart disease with PAH. ${ }^{35}$ Recently, Farinacci 
Table 2 Comparing advantages and limitations of EMB vs fluid-based assays in CV diseases

\begin{tabular}{|c|c|}
\hline Advantages & Limitations \\
\hline \multicolumn{2}{|c|}{ EMB } \\
\hline $\begin{array}{l}\text { Detailed information on cardiac tissue architecture, including myocardial cell death, } \\
\text { scars, fibrosis, disarrays, cardiomyocyte changes, pathological vascular conditions, } \\
\text { granulomas and inflammatory cell differentiation } \\
\text { Possibility of histological and molecular analysis } \\
\text { Clinical validation } \\
\text { Conclusive diagnosis of causative reasons for many CV diseases, eg, myocarditis and } \\
\text { dilated cardiomyopathy }\end{array}$ & $\begin{array}{l}\text { Sample size inadequacy and biopsy artefacts } \\
\text { Interpretative mistakes on behalf of pathologists } \\
\text { Limited to focal biopsy area at one point time (mainly limited to RV) } \\
\text { Failure to reflect tissue heterogeneity } \\
\text { Invasive and costly technique } \\
\text { Associated with pain and risk for patients } \\
\text { Difficult to repeat (not ideal for monitoring disease and response to drugs over } \\
\text { time) }\end{array}$ \\
\hline \multicolumn{2}{|r|}{ Fluid-based assays } \\
\hline $\begin{array}{l}\text { Isolation of intact cells and many biological molecules from many biological fluids } \\
\text { Mirror of cardiac tissue heterogeneity and dynamics } \\
\text { Sources of non-invasive biomarkers } \\
\text { Less expensive, quick and easily repeatable tests } \\
\text { Minimal pain/risk } \\
\text { Potential early diagnosis } \\
\text { Potential real-time monitoring of CV disease progression and individual drug response }\end{array}$ & $\begin{array}{l}\text { Lack of standardised protocols } \\
\text { Management of small amounts and easily degradable materials after harvesting } \\
\text { Need to extremely sensitive and accurate tests } \\
\text { Absence of clinical validation }\end{array}$ \\
\hline
\end{tabular}

$\mathrm{CV}$, cardiovascular; EMB, endomyocardial biopsy; RV, right ventricle.

et $a l^{20}$ have established a robust flow cytometric assay for CEC and EPC quantification in 101 patients affected by HF, diabetic nephropathy (DN) and hypertension (HT) compared with 11 controls. Evidence from this study reported that increased CEC counts may be a reliable diagnostic biomarker for DN and $\mathrm{HF}$ with preserved ejection fraction $(\mathrm{EF}){ }^{20}$ Another case-control study evaluated a correlation between the percentage of CECs and levels of endothelin-1 (ET-1) in peripheral whole blood isolated from 15 left-to-right shunt CHD without PAH, $26 \mathrm{CHD}$ complicated with mild $\mathrm{PAH}$ and $17 \mathrm{CHD}$ complicated with moderate-to-severe $\mathrm{PAH}$ with respect to 30 controls. $^{42}$ From results, an increased CEC number causing ET-1 production was observed in patients with CHD-PAH, suggesting that a combination of CECs and ET-1 may be used to define therapeutic strategies for control of PAH onset. ${ }^{42}$ Moreover, Watt et $a l^{43}$ reported that EPC count positively correlated with plasma oxidised lowdensity lipoprotein levels and coronary endothelial dysfunction in patients with stable CHD treated with standard pharmacotherapy for $\mathrm{CHD}$, including a high prevalence of statins. The authors also emphasised a putative statin-mediated mechanism

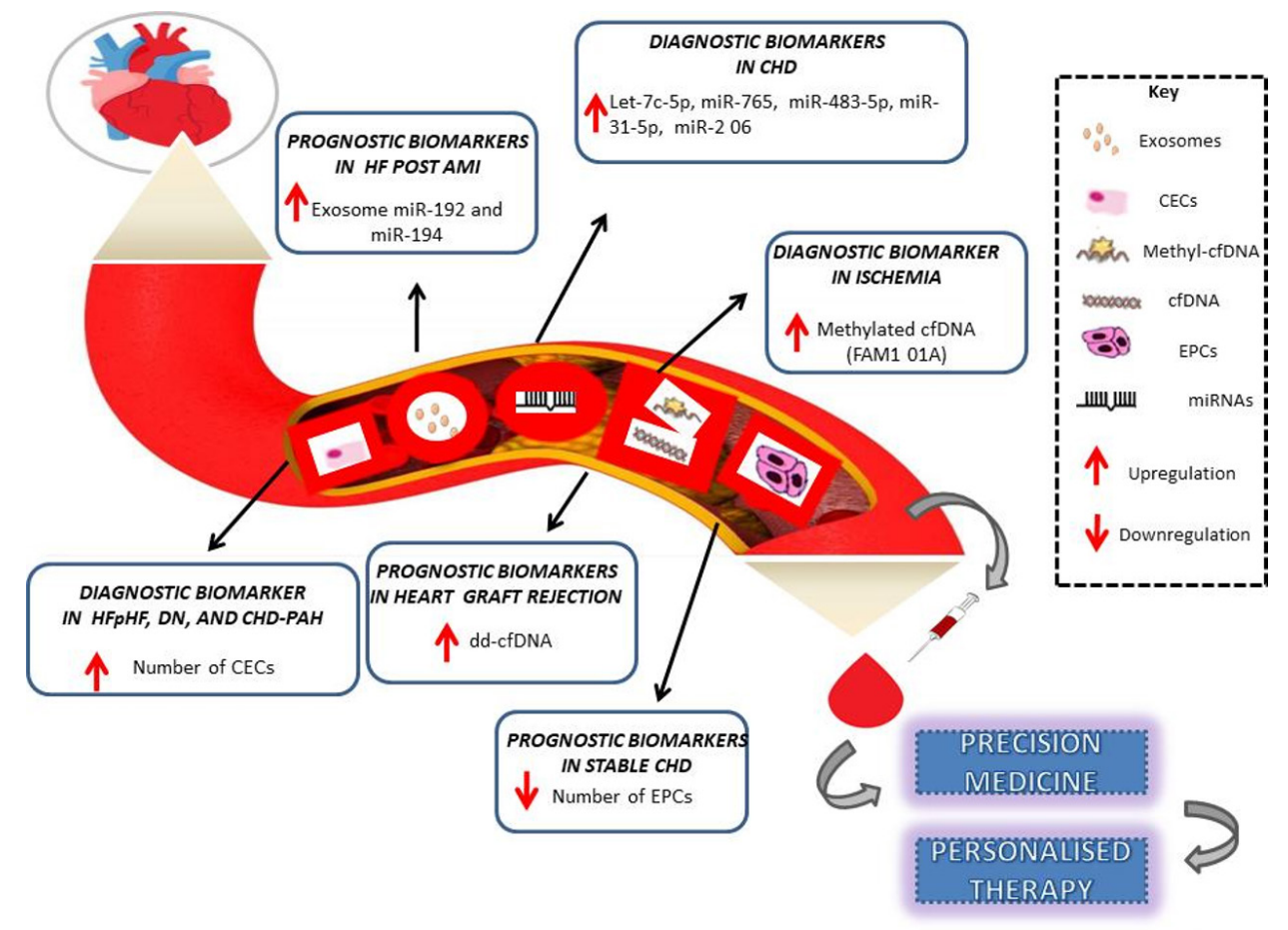

Figure 2 Some clinical applications of blood-based liquid biopsy in CV diseases. The picture illustrates the putative clinical role of some circulating non-invasive biomarkers in patients affected by common CV diseases. These molecular changes may be the most fruitful way to reach precision medicine in CV clinical setting. AMI, acute myocardial infarction; CECs, circulating endothelial cells; CHD, coronary heart disease; CV, cardiovascular; cfDNA, circulating cell-free DNA; dd-cfDNA, donor-derived cfDNA; dn, diabetic nephropathy; EPCs, endothelial progenitor cells; FAM101A, refilin a; $\mathrm{HFpEF}$, heart failure preserved ejection fraction; miRNA, micro-RNA; PAH, pulmonary arterial hypertension. 
of host-response repair to endothelial injury suggesting that epigenetic-sensitive changes should be further investigated about the putative role of EPCs in cardiac regenerative medicine. ${ }^{43}$

\section{Cell-free DNA}

Physiologically, low levels of fragmentised nucleosome-size cell-free DNA (cfDNA) can briefly circulate in the plasma of healthy subjects before being cleared by the liver. cfDNA is mainly derived from cell apoptosis ${ }^{44}$ and mostly from blood cells. ${ }^{45}{ }^{46}$ In the most cases, cfDNA is simply a product of cell death; however, its diagnostic value is certainly high in specific $\mathrm{CV}$ diseases. Indeed, under pathological conditions leading to its overproduction, larger amounts of cfDNA can be detected in the circulation suggesting a putative clinical role in stroke, AMI and allograft transplant rejection.

A case-control study reported that baseline circulating cfDNA levels were higher in 54 patients with acute ischaemic stroke receiving tissue plasminogen activator recombinant with respect to controls. ${ }^{21}$ Another group demonstrated that the mean concentration of plasma circulating cfDNA was fivefold higher in 160 patients with AMI compared with 30 controls. ${ }^{47}$ Moreover, a longitudinal monitoring of patients with AMI revealed a strong association between levels of cfDNA and severity of disease state suggesting a useful prognostic biomarker for developing of reinfarction or $\mathrm{HF}^{47}$ A prospective cohort study (21 paediatric and 44 adult patients undergoing heart transplantation tested the clinical utility of plasma donor-derived cfDNA (dd-cfDNA) levels in measuring acute graft rejection. ${ }^{22}$ The authors used the prior performed 'genome transplant dynamics' (GTD) technique based on single nucleotide polymorphisms differences between DNA molecules belonging to the recipient and donor. ${ }^{22}$ From results, patients with either acute cellular rejection or antibodymediated rejection exhibited higher dd-cfDNA levels compared with stable transplant recipients. ${ }^{22}$ Furthermore, the fraction of dd-cfDNA increased with severity of rejection and was higher up to 5 months before the biopsy-proven rejection event, suggesting dd-cfDNA as early diagnostic marker of transplant rejection. ${ }^{22}$ However, larger prospective cohort studies are needed to really confirm or not its clinical value.

\section{Circular RNAs}

Circular RNAs (circRNAs) have covalently linked ends with no polyadenylated tails and act as RNA-binding agents, sequestering agents, transcriptional regulators as well as micro-RNA (miRNA) sponges. ${ }^{48}$ Several piece of evidence report that circRNAs may play important roles in HF, CHD and AMI onset. ${ }^{48}$ By combining proteomic screening, bioinformatics and functional studies, a cross-sectional cohort study (LIFE Heart Study) demonstrated that higher levels of antisense non-coding RNA in the INK4 locus (circANRIL) were present in human atherosclerotic plaques. ${ }^{49}$ Physiologically, circANRIL can regulate ribosomal RNA maturation resulting in the induction of apoptosis and inhibition of proliferation conferring atheroprotection in humans. ${ }^{49}$ For the first time, Zhao et $a l^{50}$ measured levels of circRNA expression by using a microarray platform on peripheral blood isolated from 12 patients with CHD with respect to 12 controls and found that hsa_circ_0124644 was significantly upregulated in the CHD group, suggesting a potential diagnostic biomarker for disease. ${ }^{50}$ However, a multicentre investigation is required to validate these results. Moreover, Salgado-Somoza et $a l^{11}$ reported that myocardial infarction-associated circular RNA (MICRA) may be useful to predict the risk in patients with AMI. By using blood samples from 472 patients with AMI, they found that the expression levels of MICRA were lower in patients with $\mathrm{EF} \leq 40 \%$ with respect to patients with $\mathrm{EF}>41 \%$, thus indicating a useful predictive biomarker for post-AMI left ventricle remodelling. ${ }^{51}$ Recently, Bao et $a l^{52}$ detected that hsa_circ_0037911 levels were higher in 100 newly diagnosed essential hypertension (EH) patients with respect to 100 controls and correlated with serum creatinine $(\mathrm{Scr})$. Results from this study suggested that higher expression of hsa_circ_0037911 may be crucial for EH development by modulating Scr levels, thus providing a useful biomarker for early diagnosis of disease. ${ }^{52}$ Importantly, circRNAs present several advantages over linear RNAs. Indeed, they are more abundant and more stable (half-life of about 48 hours) than linear RNAs (eg, mRNAs, 10 hours) due to the covalently closed-loop structures that can resist RNA exonuclease and RNase R activity making them ideal biomarkers. ${ }^{53}$

\section{Circulating epigenetic signatures Methylated DNA}

A real challenge in liquid biopsy of epigenetic biomarkers is the ability to identify tissue-specific DNA methylation patterns in order to trace the source of circulating cfDNA molecules. For the first time, Sun et al developed a general strategy for tracing cfDNA using DNA methylation able to identify the major tissue contributors to the circulating DNA pool, including pregnant women, cancer and liver transplantation. ${ }^{45}$ Interestingly, a comparative methylome analysis (right atrium, left and right ventricle vs 23 other human tissues) was performed to establish putative genomic loci that are methylated in a cardiac-specific manner. ${ }^{54} \mathrm{By}$ results, the refilin A (FAM101A) gene was highly unmethylated in cardiac tissues with respect to others and was then used to assess the concentration of cardiac cfDNA in CV patients. Remarkably, higher levels of cfDNA was observed in plasma isolated from 57 patients with acute ST-elevation myocardial infarctions with respect to 83 controls, suggesting a powerful diagnostic tool for cardiomyocyte apoptosis during ischaemia. ${ }^{55}$ Of note, DNA methylation is one of the most important epigenetic links between genome and environment contributing to in utero development of early atherosclerotic lesions by modulating gene expression at transcriptional level. ${ }^{556}$ Beyond its putative diagnostic power, it is necessary to highlight that DNA methylation may serve as a reversible drug target depending on a biochemical cycle that requires methyl donors. ${ }^{57} 58$ For example, agents inhibiting DNA methylation, such as 5-aza-2-deoxycytidine, are associated with attenuation of cardiac phenotypes. ${ }^{5859}$ Thus, an individual DNA methylation map may be used to identify subjects who might benefit from personalised treatments and nutritional interventions.

\section{MicroRNAs}

miRNAs are endogenous single-stranded non-coding RNAs of 21-22 nucleotides regulating gene expression at posttranscriptional and translational level (RNA interference). These ribomolecules promote degradation or translational repression by imperfect base-pairing with the $3^{\prime}$ untranslated region of target mRNAs ${ }^{60}$ miRNAs are critical regulators of $\mathrm{CV}$ function and play crucial roles in almost all aspects of CV biology. ${ }^{61}$ Recently, Wang et $a l^{62}$ have reported elevated levels of circulating miR-92a in patients with type 2 diabetes (T2D) associated with an increased risk of acute coronary syndrome (ACS) with respect to controls. Moreover, the combination panel of glycosylated haemoglobin, systolic blood pressure and miR-92a showed a better predictive value than any individual biomarker alone, with 95\% sensitivity and $95 \%$ specificity. ${ }^{62}$ According to prior investigations, ${ }^{63}$ a large and robust study reported that a gradual reduction of some circulating 
miRNAs, including miR-18a, miR-27a, miR-30e, miR-26b, miR199a, miR-106a and miR-652 was found in acute HF compared with both patients with chronic HF and healthy controls. ${ }^{64}$ Moreover, lower levels of circulating miRNAs let-7i, miR-18b, miR18a, miR-223, miR-301a, miR-652 and miR-423 were reported within 48 hours after acute HF admission and were associated with an increased risk of 180-day mortality. ${ }^{64}$ However, multicentre studies with a large sample size are needed to validate these predictive values. For the first time, a large cohort of 953 patients with chronic HF enrolled in the multicentre Gruppo Italiano per lo Studio della Sopravvivenza nella Insufficienza Cardiaca-Heart Failure (GISSI-HF) trial demonstrated that higher circulating miR-132 levels were independently associated with younger age, better renal filtration, ischaemic aetiology of HF, more severe HF symptoms, higher diastolic blood pressure, higher cholesterol and male sex with respect to controls, suggesting a useful risk predictor for hospitalisation. ${ }^{65}$ Importantly, in two independent cohorts of 2203 patients with HF, miR-1254 and miR-1306 were associated with increased risk of death and hospitalisation. ${ }^{66}$ Beyond the putative role as predictive, diagnostic and prognostic biomarkers, all of these changes in circulating miRNA also suggest innovative therapeutic targets. ${ }^{61}$ Indeed, in recent years many miRNA mimics and anti-miRs (synthetic oligonucleotides that block miRNA function) were designed and evaluated in preclinical studies for treatment of different CV diseases by targeting molecular pathways including apoptosis, autophagy, hypertrophy. Thus, this issue deserves further investigations in clinic trials. ${ }^{61}$

\section{Modified histones}

Neutrophil extracellular traps (NETs) are circulating double strand DNA (dsDNA)-based complexes which form fibrous structures with histones and granule proteins to trap microorganisms and kills them by antimicrobial properties. ${ }^{67}$ On inflammatory stimuli, the overexpression of peptidyl arginine deiminase 4 enzyme can lead to modification of specific arginine into citrulline residues (citrullination) at the level of $\mathrm{H} 3$ and $\mathrm{H} 4$ tails inducing the expulsion of NETs from activated neutrophils. ${ }^{67}$ This process, called NETosis, plays a key role in increasing the risk of venous thromboembolism, owing to the strong capability of circulating histones to enhance generation of thrombin, secretion of Weibel-Palade body and adhesion of platelet and leucocytes. ${ }^{67}$ A prospective cohort study reported that levels of circulating histones incorporated into NETs significantly increased in congenital cardiac patients with adverse events immediately after cardiopulmonary bypass (CPB) with respect to controls. ${ }^{6}$ Importantly, circulating histones reached the maximum value earlier than $\mathrm{N}$-terminal probrain natriuretic peptide, procalcitonin and $\mathrm{C}$ reactive protein (CRP) after $\mathrm{CPB}$, suggesting a useful prognostic indicator of adverse events after cardiac surgery. ${ }^{68}$ Moreover, it has been reported that circulating cell-free dsDNA concentration may reflect the amount of NET formation in the blood and, thus, the severity of renal dysfunction in 87 patients undergoing cardiac surgery with respect to controls. ${ }^{69}$ Taken together, these results suggest that NETs may be novel biomarkers for postoperative risk assessment of patients with cardiac surgery. ${ }^{68} 69$ However, further large-scale prospective studies will be needed to illuminate the potential pathophysiological mechanisms that might be responsible of NET release in postoperative conditions.

Among histone modifications, acetylation is the most studied both as biomarker and drug target in CV diseases. This chemical modification can alter specific lysine residues on the aminoterminal tails of $\mathrm{H} 3$ and $\mathrm{H} 4$ histones playing a crucial role in chromatin structure modelling and gene transcription. ${ }^{70}$ Histone acetylation/deacetylation balance is maintained by histone acetyl transferases and histone deacetylases (HDACs) enzymes which add and remove acetyl groups, respectively. ${ }^{70}$ It has been reported that HDAC inhibitors (HDACi), such as tricostatin A, ributyrin and valproic acid show anti-inflammatory properties at low concentrations and may be also beneficial for minimising the scar size of myocardial infarction. ${ }^{71}$ Statins, as HDACi, represent a heterogeneous group of 3-hydroxy-3-methylglutaryl coenzyme A reductase inhibitors largely used as cardioprotective agents both as primary and secondary prevention. ${ }^{72}$ In particular, statins may trigger the expression of endothelial nitric oxide synthase and thrombomodulin via activation of the lung Krüppel-like factor ( $L K L F / K L F 2)$ gene promoter, providing a novel molecular target to modulate endothelial function in $\mathrm{CV}$ diseases. ${ }^{73}$ However, the debate about the use of statins is still open owing to evidence of a small increased risk of T2D in CV patients treated with this medication. ${ }^{74}$

\section{Extracellular vesicles}

Extracellular vesicles (EVs) comprise a large spectrum of organelles released from various cell types classified into exosomes, microvesicles and apoptotic bodies according to their size, cellular origin, content or the mechanism leading to their formation. ${ }^{75}$ In detail, microvesicles $(100-1000 \mathrm{~nm})$ are generated by the direct outward budding of cell membranes, whereas exosomes $(30-150 \mathrm{~nm})$ are generated by the traditional endosomal pathway playing a key role for long distance intercellular signals. ${ }^{75}$ Owing to their capability of transferring proteins and nucleic acids such as angiogenic, prosurvival factors and miRNAs from one cell to another, EVs are becoming attractive biomarkers and drug targets of CV diseases. $^{76-78}$ Interestingly, it was reported that increased expression of miR-126 and miR-199a in circulating microvesicles, but not freely circulating miRNAs, showed a cardioprotective effect by reducing risk of CV events in 187 patients with $\mathrm{CHD}$ with respect to controls. ${ }^{79}$ Moreover, Yang et $a l^{80}$ demonstrated that 145 patients with AMI had increased serum levels of exosomerelated miR-30a with respect to controls responsible for autophagy of cardiomyocytes, thus suggesting a new putative strategy to treat ischaemic heart disease. Furthermore, Matsumoto et al $l^{81}$ found that patients with AMI developing HF have elevated serum levels of exosome-related miR-192, miR-194 and miR-34a with respect to controls suggesting of a putative panel of prognostic biomarkers useful to physicians in designing more customised treatments. Remarkably, some evidence highlighted that administration of induced pluripotent stem cells (iPSC) and iPSC-derived cardiomyocytes releasing exosomes to damaged tissues may be a frontier therapy in cardiac regenerative medicine. ${ }^{82-84}$ In detail, these 'nanoshuttles' may regulate gene expression of target cells by realising specific miRNAs aimed at attenuating cardiac fibrosis and stimulating angiogenesis. ${ }^{82-84}$ However, even if administration of exosomes seem to be an excellent therapeutic tool for cardioprotection or regeneration of the injured myocardium, we are so far from clinical confirmations. ${ }^{82-84}$

\section{Circulating metabolites}

Several metabolic dysregulations occurring in cardiomyocytes as well as in other tissues were associated with a large spectrum of CV diseases. ${ }^{85}$ The human metabolome refers to the complete set of small molecules including endogenous metabolites (eg, amino acids, organic acids, nucleic acids, fatty acids, amines, sugars, vitamins, co-factors, pigments, antibiotics, etc) and exogenous chemicals (such as drugs, environmental contaminants, food additives, toxins and other xenobiotics) as products of metabolic reactions 
catalysed by numerous enzymes occurring within cells. ${ }^{86}$ All of these metabolites can be measured by using different advanced metabolomics platforms including nuclear magnetic resonance (NMR spectroscopy), mass spectrometry (MS), liquid chromatography (LC), gas chromatography or capillary electrophoresis to facilitate compound separation. ${ }^{85}$ Basic findings from metabolomics applications are contributing to better clarifying the metabolic unbalance that occurs in $\mathrm{CV}$ diseases suggesting useful non-invasive circulating biomarkers. Moreover, Würtz et $a l^{87}$ conducted NMR metabolomics to three large cohorts in order to identify circulating biomarkers of incident CV events. From these results, four metabolites were strongly associated with onset of CV diseases during the 15 years follow-up: phenylalanine, monounsaturated fatty acids, omega- 6 fatty acids and docosahexaenoic acid, suggesting their putative useful incorporation along well-established prediction models. $^{87}$ By using MS-HPLC platforms, Ganna et al ${ }^{88}$ performed a 10-year follow-up of 1028 subjects revealing that plasma profiling of lipid fractions, glucose, valine, ornithine, glutamate, creatinine, glycoproteins, citrate and 1,5-anhydrosorbitol were independent predictors of CHD onset. ${ }^{88}$ More recently, by applying MS platforms to obtain metabolomic profiling of three independent cohorts study (total $n=3924$ ), an association between circulating levels of the haem breakdown products urobilin and sphingomyelin (30:1) with incident HF suggested useful noninvasive predictive biomarkers. ${ }^{89}$ Since metabolomics deals with the end products of gene expression, this field may strongly clarify the relationship between genetic variations, environmental factors and CV diseases. Thus, further longitudinal studies may reveal novel metabolic pathways and biomarkers for improving personalised therapy of CV diseases.

\section{A focus on saliva and urine liquid biopsy in CV diseases}

Over the last decade, human saliva has attracted attention as a liquid biopsy for prevention and diagnosis of some $\mathrm{CV}$ diseases. ${ }^{90-93}$ Saliva collection is non-invasive, convenient and inexpensive compared with conventional venipuncture procedure showing a promising potential as a diagnostic fluid. Salivary proteome may be useful to trace inflammatory state in CV patients. ${ }^{90-93}$ Miller et $a l^{93}$ established that salivary MYO levels were higher within 48 hours of the onset of angina in 92 patients with AMI with respect to controls suggesting a useful role in combination with ECG for the identification of disease state. Moreover, a strong link was reported between increased levels of serum/salivary creatine phosphokinase MB and creatine phosphokinase (CPK) at onset of 12 and 24 hours of patients with AMI compared with controls. ${ }^{83}$ Labat et al ${ }^{94}$ collected saliva and plasma samples from 250 individuals with a history of CV events and demonstrated that salivary levels of CRP, prostaglandin E2, leukotriene B4, matrix metalloproteinase-9, creatinine and lysozyme may be alternative biomarkers for evaluation of CV risk. It is well known that the above-mentioned biomarkers are general and non-specific, thus major efforts should be done to identify a panel of more specific indicators so that saliva become a reliable diagnostic fluid for CV diseases. Also, human urine sampling is being tested as a putative fluid-based strategy revealing a large number of peptides as accurate biomarkers for early asymptomatic stages of CV diseases. ${ }^{95-97}$ Recently, Bazzell et al ${ }^{95}$ demonstrated that RNA-sequencing profiles of human urinary EVs and renal cortex tissue were similar and changes in mRNA levels in urine supernatant (US-mRNA) could reflect renal gene expression in resistant hypertension. This evidence supports the idea of using US-mRNA to detect changes in renal or cardiorenal physiology suggesting a novel non-invasive diagnostic test for
CV diseases. ${ }^{95}$ Further studies are needed to determine if urine liquid biopsy may be translated in CV clinical practice.

\section{FLUID-BASED ASSAYS IN CLINICAL TRIALS OF CV DISEASES}

Despite great efforts into developing new techniques, so far liquid biopsy strategies are not implemented in routine $\mathrm{CV}$ clinical. Moreover, evidence-based guidelines indicating effective pharmacological effects are also lacking. One reason is that efforts in clinical translation of basic findings is still in its infancy, as demonstrated by the small number of large randomised trials completed in the last 10 years. We systematically searched https:/clinicaltrials.gov/ website for liquid biopsy studies in CHD, AMI, HF and PAH by using criteria that blood, saliva and urine were included as a biological sample and preliminary results were published. Based on these criteria, seven clinical trials were included: four interventional, randomised, two observational, prospective and one observational, retrospective (table 3). In order to highlight the increasing interest in this field, we discuss some of these results. The Surveillance HeartCare Outcomes Registry is an ongoing clinical trial (NCT03695601) evaluating the fraction of plasma dd-cfDNA discriminating unstable from stable transplant recipients. Preliminary data reported that the percentage of dd-cfDNA, measured by unbiased shotgun assay, is precise and reproducible across different laboratories and patient cohorts suggesting its putative clinical value as non-invasive biomarker of acute rejection after heart transplantation. A whole-genome miRNA sequencing was performed on RNA extracted from whole blood of 199 patients with non-STsegment elevation ACS (NSTE-ACS) and controls belonging to the TaRgeted platelet Inhibition to cLarify the Optimal strateGy to medicallY manage ACS trial (NCT00699998) to find novel putative biomarkers improving risk stratification in patients who experience ACS events. From this, miR-126-5p, miR-142-5p, miR-144-5p, miR-28-3 p and miR-3135b are significantly associated with chronic HF and the Global Registry of Acute Coronary Events risk score, suggesting novel putative predictors of mortality in patients with NSTE-ACS. Furthermore, by using a subgroup of the Target Temperature Management randomised trial (NCT01020916), it was reported that higher circulating miRNA-124-3p levels may be putative useful indicators of poor neurological outcomes and survival after out-of-hospital cardiac arrest, as well as innovative drug targets for tailored management. Moreover, EPCs are in double-blind, randomised, placebo-controlled phase IV clinical trial (NCT02194686 and NCT01096875) to evaluate the effects of cilostazol and atorvastatin, as HDACi, in patients with high risk for $\mathrm{CV}$ diseases. Although promising results, none of these indicators is used in CV clinical setting. More large prospective study population are needed as well as the implementation of innovative research programme in clinical trials (discussed in the 'Challenges and opportunities for clinical trials from big data consortia' section).

\section{BIG DATA AND NETWORK MEDICINE: NOVEL INTEGRATED APPROACHES FOR CV DISEASES}

\section{Why big data are important in the modern CV medicine?}

Healthcare big data refer to collecting, analysing and leveraging consumer, patient, physical and clinical data that are too vast or complex to be understood by traditional means of data processing. Instead, big data are often processed by machine learning algorithms and data scientists. ${ }^{98}$ The rise of healthcare big data comes in response to the digitisation of 


\begin{tabular}{|c|c|c|c|c|c|c|c|}
\hline NCT & Study type & Participants & Conditions & Circulating markers & Phase & Aims & Reference \\
\hline NCT03695601 & $\begin{array}{l}\text { Observational, } \\
\text { prospective }\end{array}$ & 1600 & $\begin{array}{l}\text { Heart transplant } \\
\text { rejection }\end{array}$ & dd-cfDNA & $\mathrm{N} / \mathrm{A}$ & $\begin{array}{l}\text { To monitor heart transplant recipients for } \\
\text { allograft rejection. }\end{array}$ & 115 \\
\hline NCT01020916 & $\begin{array}{l}\text { Interventional, } \\
\text { randomised }\end{array}$ & 950 & $\begin{array}{l}\text { Out-of-hospital } \\
\text { cardiac arrest }\end{array}$ & miRNA-124-3p & $\mathrm{N} / \mathrm{A}$ & $\begin{array}{l}\text { To evaluate a target temperature management } \\
\text { after cardiac arrest. }\end{array}$ & 116 \\
\hline NCT02299960 & $\begin{array}{l}\text { Observational, } \\
\text { prospective }\end{array}$ & 101 & $\begin{array}{l}\text { Heart failure, } \\
\text { hypertension, } \\
\text { pulmonary } \\
\text { hypertension, } \\
\text { diabetic } \\
\text { nephropathies }\end{array}$ & CECS & $\begin{array}{l}\mathrm{N} / \mathrm{A}, \\
\text { completed }\end{array}$ & $\begin{array}{l}\text { To correlate analyse test-retest reliability } \\
\text { (EndoPAT), circulating endothelial cells and } \\
\text { and endothelial function in CV patients. }\end{array}$ & 20 \\
\hline NCT00699998 & $\begin{array}{l}\text { Interventional, } \\
\text { randomised }\end{array}$ & 9326 & $\begin{array}{l}\text { Acute coronary } \\
\text { syndrome }\end{array}$ & miRNAs & $\begin{array}{l}\text { Phase III, } \\
\text { completed }\end{array}$ & $\begin{array}{l}\text { To assess the efficacy and safety of novel } \\
\text { combination therapy and useful biomarkers. }\end{array}$ & 62 \\
\hline NCT02325765 & $\begin{array}{l}\text { Observational, } \\
\text { retrospective }\end{array}$ & 100 & $\begin{array}{l}\text { Congential cardiac } \\
\text { surgery }\end{array}$ & Histones & Completed & $\begin{array}{l}\text { To determine the correlation between } \\
\text { circulating histones and the development of } \\
\text { SIRS. }\end{array}$ & 68 \\
\hline NCT02194686 & $\begin{array}{l}\text { Interventional, } \\
\text { randomised }\end{array}$ & 71 & CV diseases & EPCs & $\begin{array}{l}\text { Phase IV, } \\
\text { completed }\end{array}$ & $\begin{array}{l}\text { To evaluate the effects of cilostazol on human } \\
\text { early EPCs and endothelial function. }\end{array}$ & 117 \\
\hline NCT01096875 & $\begin{array}{l}\text { Interventional, } \\
\text { randomised }\end{array}$ & 60 & $\begin{array}{l}\text { Coronary artery } \\
\text { bypass surgery, } \\
\text { elective surgical } \\
\text { procedure }\end{array}$ & EPCs & $\begin{array}{l}\text { Phase IV, } \\
\text { completed }\end{array}$ & $\begin{array}{l}\text { To test if atorvastatin augments the number of } \\
\text { EPCs after cardiopulmonary bypass. }\end{array}$ & 118 \\
\hline
\end{tabular}

CECs, circulating endothelial cells; CV, cardiovascular; dd-cfDNA, donor-derived cell-free DNA; EPCs, endothelial progenitor cells;miRNA, micro-RNAs; N/A, not applicable; SIRS, systemic inflammatory response syndrome.

healthcare information and the rise of value-based care, which has encouraged the industry to use data analytics to make strategic business decisions. ${ }^{98}$ Faced with the challenges of healthcare data, such as volume, velocity, variety and veracity, health systems need to adopt technology capable of collecting, storing and analysing this information to produce actionable insights. ${ }^{98}$ One of the biggest hurdles standing in the way to use big data in medicine is how medical data are spread across many sources governed by different states, hospitals and administrative departments. Integration of these data sources would require developing a new infrastructure where all data providers collaborate with each other. ${ }^{98}$ One example of big data application is the use of HER, digital record for each patient, which includes demographics, medical history, allergies, laboratory test results, etc. ${ }^{2}$ Records are shared via secure information systems and are available for providers from both public and private sector. Every record comprises one modifiable file, which means that doctors can implement changes over time with no paperwork and no danger of data replication. Moreover, big data can be integrated with imaging: algorithms analysing hundreds of thousands of images could identify specific patterns in the pixels and convert it into a number to help the physician with the diagnosis. ${ }^{99}$ Indeed, Carestream (https://www.carestream.com), a medical imaging provider, explains that it could be possible that radiologists will no longer need to look at the images but instead analyse the outcomes of the algorithms that will inevitably study and remember more images than they could in a lifetime. In the CV field, the ability to convert analogue data into digital data (the entire dataset is numeric, the image is a rendering) is demonstrated, for example, by generation of the ' $\mathrm{CHADS}_{2}$ ', a simple scoring system that uses five common stroke risk factors: congestive heart failure, hypertension, age $>75$ years, diabetes (all one point each); previous stroke (two points) derived from the combination of two separate risk schemas based on the historical trials of Stroke Prevention in Atrial Fibrillation (SPAF) and the SPAF-1 trial and subsequently validated in a registry of hospitalised non-valvular patients with AF. ${ }^{100}$

\section{Network medicine}

In the era of network medicine, customised therapy for $\mathrm{CV}$ diseases involves a combination of HER, imaging and multiple, advanced 'omics' tools focusing on genomics, transcriptomics, epigenomics, proteomics, metabolomics as well as dietary habits (foodome) and environmental exposures. ${ }^{2}$ 7-9 1214 The key concept of network medicine is that a disease can result from a chain of perturbations in the human interactome rather than a singular defect in a candidate gene, as emphasised in the current reductionist approach to drug development. ${ }^{2}$ 7-9 1214 For example, hypertrophic cardiomyopathy (HCM) has been ascribed to single sarcomere gene mutations from reductionism; however, these gene abnormalities did not explain the overall HCM clinical and pathobiological features. ${ }^{38}$ Thus, networkoriented analyses may unravel the pathobiological complexity of disease. ${ }^{38}$ Network-based analyses allow for identification of complex molecular perturbations in which disease genes or gene products are clustered in discrete disease modules in the interactome (disease modules or subnetworks). ${ }^{27-9} 1214$ In each module disease, a molecular network is a set of points (nodes) that are linked in pairs by lines (edges). Nodes can represent genes, proteins and metabolites, whereas edges represent physical or functional relationships among them leading to a path visualised and analysed by using graph theory. ${ }^{2}$ 7-9 1214 The starting points for network analyses are big data integrated in multilayered datasets, for example, genome-wide association studies (GWASs) and literature. To date, the huge amount of big data is collected and updated in open-to-public databases, such as GEO, KEGG, DisGeNET, STRING, which integrates information on gene-disease associations from various public repositories and the biomedical literature..$^{27-9} 1214$ In particular, CardioVINEdb is a user-friendly independent web interface that can be used with any common web browser. ${ }^{101}$ This one catalogues protein-protein interactions (PPIs), metabolic and regulatory pathways providing a static/dynamic visualisation for network components and an interactive graphical exploration of the data. ${ }^{101}$ This kind of datasets represents the start point 
to capture crucial nodes underlying the changes in gene expression associated with CV diseases based on their topological position in the human interactome. However, these data are simply too massive for a human to rapidly process, or process at all. How could we analyse the comprehensive big datasets for meaningful insights into CV pathobiology? Network analyses offer a broad panel of quantitative algorithms including PPIs networks, in which the nodes are proteins linked by physical/functional interactions (eg, GenePanda, DIAMOnD, PRINCE, ProDiGe and DADA), regulatory networks, thereby the links represent regulatory relationships between a transcription factor and its target gene (eg, PANDA) and co-expression networks, in which the nodes are represented by mRNAs and a link occurs if their expression profiles are either highly correlated or anticorrelated (eg, SWIM and WGCNA). ${ }^{27-9} 1214$ In literature, there are several examples in which the application of network-based analyses has improved pathobiological knowledge of conventionally defined CV diseases leading to a putative future molecular-based system to redefine diseases with respect to the conventional approach used for diagnosis, mainly focused on physiology and/ or pathology. ${ }^{7-9} 121438$ Nowadays, the main goal of network medicine is to identify and validate disease-related interaction networks to provide novel specific biomarkers able to predict high-risk subjects, diagnose early asymptomatic stage of disease, monitor patient follow-up and predict response to drug therapy. ${ }^{27-9} 1214$ In particular, researchers can verify if the predicted disease module really exists by perturbing it through pharmacological (eg, RNA interference) or genetic (CRISPR/ Cas9) strategies. As confirmed by computational findings, these perturbations should lead to a change in the phenotype. ${ }^{102}$ Even if numerous valid models exist to address validation of target nodes or pathways, to date we are far from translation of basic findings in clinical market. Also, network medicine offers an alternative approach to drug discovery in CV diseases focusing on identification of molecular network perturbations at an individual level and development of drugs that can affect the molecular pathways rather than only a single protein. ${ }^{13}$ Recently, a network-based pharmacology approach for target identification and drug repurposing has been recently performed suggesting that several approved drugs (eg, fasudil, parecoxib and dexamethasone) or natural products (eg, resveratrol, luteolin, daidzein and caffeic acid) may be useful in treatment and prevention of CHD. ${ }^{103}$

\section{How could we integrate fluid-based assays with network medicine?}

Epigenetic-oriented liquid-based assays and imaging

The ability to design customised treatments relies strongly on the possibility of predicting the course of the disease in individual $\mathrm{CV}$ patients. Epigenetic-sensitive molecular mechanisms are crucial to understand how sensitiveness to cardiac phenotypes changes over time and potent imaging tools, such as cardiac computed tomography (CTT), combined with circulating miRNAs (upregulation of let-7c-5p, miR-765, miR-483-5p, miR-31-5p and miR-206) provides the framework to construct risk predictive models in CHD. ${ }^{104}$ Another epigenetic/imaging approach useful to support the diagnosis and prediction of CHD combines the methylation status of specific cholesterol metabolism loci, including low-density lipoprotein receptor, sterol regulatory element-binding factor 2 (SREBF2) and ATP-binding cassette transporter 1 ( $A B C A 1)$ genes in circulating peripheral blood mononuclear cells and CCT analysis. ${ }^{105}$ These epigenetic changes are crucial in the early steps of human atherogenesis. ${ }^{55}$
Results demonstrate higher levels of DNA methylation at SREBF2 and ABCA1 gene promoters in patient with CHD and obstructive CHD. ${ }^{105}$ More extensive analysis of $\mathrm{CV}$ phenotypes and circulating biomarkers might improve and personalise $\mathrm{CV}$ risk stratification in the clinical setting.

\section{The importance of fluid-based assays to identify PPIs network} biomarkers in longitudinal clinical trials

Whereas most clinical trials measure systemic levels of one or a few static indicators of disease alterations or severity, networkbased approaches are testing gene-gene interactions and relative biomarkers in prediction of CV clinical outcomes. ${ }^{106-108}$ PPIs play a critical role in many biological functions by mediating the signalling pathways. It was found that PPI network biomarkers discovered by proteomics platforms were better than single biomarkers without any consideration of interaction in classification of CV patient with acute events. ${ }^{109}$ To shift the attention from static to dynamic properties of the interactome, dynamical PPI networks (DPINs) biomarkers were constructed by focusing on information extracted from gene expression data in different time points. ${ }^{110}$ Whereas network biomarkers highlight the interaction among molecules, DPINs focus on dynamical alterations of biomarkers to provide a more accurate picture for biomarkers mining during disease development. Indeed, DPINs show spatiotemporal alterations that are monitored and evaluated at different stages and time points during disease occurrence, progression or treatment. ${ }^{110}$ Moreover, it was proposed that DPINs may identify a predisease state even with big data and are delivered by small amounts of samples. ${ }^{111}$ Why liquid biopsy strategies needed to discover novel DPINs for CV diseases? Remarkably, liquid biopsy can compensate for the limitations of EMB by providing the most dynamic source of information about a large spectrum of molecular signatures varying with time and localisation. On the other hand, network medicine can increase the performance of fluid-based assays strategies by addressing some of the current limitations. In particular, network medicine may offer a framework for the future clinical validity, and, most importantly, clinical utility of fluid-based assays in CV field. Indeed, network medicine offers a more holistic perspective of $\mathrm{CV}$ diseases pointing to correlate complex molecular perturbations with clinical data such as clinical symptoms and signs, physician examinations, biochemical analyses, imaging profiles, history, therapies and other measurements. ${ }^{2-9} 1214$ Thus, combination of fluid-based assays and network-oriented analyses may aid to design prospective studies to obtain predictive models of evolution of CV diseases, with major emphasis on predisease state, enabling physicians to anticipate cardiac dysfunction and modulate therapeutic decisions (figure 3).

\section{Challenges and opportunities for clinical trials from big data consortia}

Integrating fluid-based assays with network-oriented analyses in longitudinal clinical trials is an ambitious project that might be realisable because of a large number of data repositories and browsers that are currently available thanks to the endeavours of big data consortia. For example, the Biomarker for Cardiovascular Risk Assessment across Europe (BiomarCaRE) consortium is an European Union-funded consortium including over 30 partners from academia and industry, unique for its dimension and goals. BiomarCaRE aims to determine the value of established and emerging omics-based biomarkers to improve CV risk estimation in Europe. ${ }^{112}$ BiomarCaRE relies on an exceptional resource of large-scale epidemiological cohorts with long-term 
A

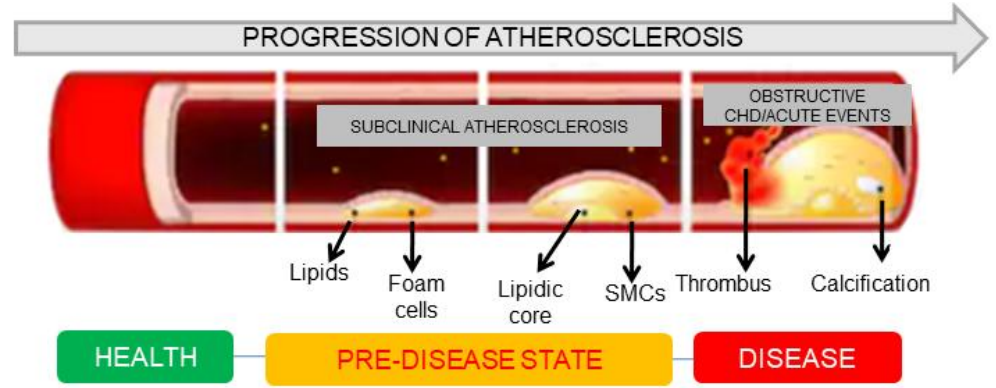

PRIMARY PREVENTION OF CHD

Figure 3 Atherosclerosis progression, dynamical network biomarkers and liquid biopsy. (A) Progression from normal to predisease state and conclamate disease represents the evolution model of atherosclerotic plaques over time. Generally, the healthy state is characterised by a chronic inflammation during which the disease is under control, whereas a predisease state is the limit of the normal state just before the critical transition into the conclamate disease occurring with thrombus formation and calcification. (B) Integrated approaches combining liquid biopsy, omics platforms and network analyses can provide novel putative useful DPINs, which are network biomarkers showing time-dependent alterations when monitored and evaluated at different stages and time points during the disease development. Remarkably, DPINs may detect early signals of the endothelial damage, known as 'predisease state', providing useful indicators to prevent the critical transition from normal to conclamate CHD and achieve the early diagnosis and intervention for acute or obstructive events. CHD, coronary heart disease; DPINs, dynamical protein interaction networks; SMCs, smooth muscle cells.

follow-up and available biospecimens based on the population of the MOnica Risk, Genetics, Archiving and Monograph (MORGAM) Project as well as several CV disease cohorts and clinical trials. ${ }^{112}$ Importantly, all epidemiological and clinical phenotypes as well as disease outcomes have been harmonised in a joint database. The design of clinical trials in the BiomarCaRE shows a multimodular concept: 1) biomarker selection and assay development, 2) biomarker measurements and statistical analyses and 3) clinical translation and economic assessment. ${ }^{112}$ In detail, established and emerging omics-based biomarkers are prioritised according to their correlation with CV risk and new indicators are selected based on pre-existing non-publicly available omics datasets. The development of molecular assay is guided by small-to-medium enterprise and optimised for mediumthroughput to high-throughput measurement. The predictive value of biomarkers is assessed separately in population and CV diseases-based cohorts in a two-phase approach: phase I assessment and phase II validation. Finally, the third module assesses the clinical utilisation of BiomarCaRE risk panels in randomised clinical trials for their interaction with risk-lowering therapy and develops a decision analytical model to estimate long-term cost-effectiveness of a primary or secondary preventive strategy guided by biomarker testing. ${ }^{112}$ In this dimension, the use of potent bioinformatic approaches, including networkoriented analyses, may aid to prioritise huge molecular pathways which may provide useful preventive, diagnostic or therapeutic targets, thus accelerating the translation in CV clinical practice.
However, standardisation of protocols, time and costs are some of the main current limitations for this objective.

\section{DISCUSSION}

Currently, liquid biopsy is not yet considered a standard testing procedure but it is used primarily as a complimentary strategy in oncology, where there is a major interest in the field. Despite a global increase in the performance of techniques, lack of standardised procedures, management of small amount of materials, challenges for test specificity and sensitivity are current limitations of published protocols (online supplementary table 1). Indeed, NGS, flow cytometry, real-time PCR (RT-PCR), ELISA, NMR and MS show remarkable differences for identifying false positive and negative results. Certainly, pre-analytical variability, extraction and detection modalities and normalisation processes should be improved but, importantly, an optimal workflow for bioinformatic analyses (eg, network algorithms) is also required for further integration and optimisation such that liquid biopsy can be routinely used in the clinic of CV diseases. Another limitation that we noted is the absence of performance comparison between liquid biopsy and EMB for the most of studies reviewed. Indeed, this was performed only in the study focused on cfDNA as prognostic biomarker of heart transplantation. ${ }^{22}$ Interestingly, results from GTD platform demonstrated a superior performance when compared with EMB, as well as the AlloMap test, a commercial gene expression assay that is currently available for the noninvasive monitoring for rejection after heart transplantation. ${ }^{113}$ 
Among the reviewed studies, the information obtained from Wang et $\mathrm{l}^{62}$ reported the higher sensitivity (85\%) and specificity $(82 \%)$ in predicting ACS events by using RT-PCR to assess levels of circulating miR-92 in plasma of patients with diabetes. Despite limitations due to miRNA isolation procedures, types of sample and study methodology (single-centre vs multicentre study), these molecules are remarkably stable and detection is reproducible, thus representing useful and early biomarkers for CV disease diagnosis, prevention and prognosis. However, further validation in clinical trials is required to support the utility of this test.

Furthermore, miRNA-based CV therapeutic strategies hold a great clinical potential, owing to the ability to deliver oligonucleotides to mimic miRNA expression or to employ small molecules to increase or inhibit miRNA function. Also, more efficient and selective in vivo delivery systems are needed to minimise the risk of unwanted side effects arising from a systemic miRNA delivery.

Several limitations also arise from various metabolomics platforms because metabolite assessment is often semiquantitative rather than quantitative, making it difficult to compare and combine results among different studies and to determine the levels for practical use. Some clinical trials provided positive results for liquid biopsy of cfDNA as a prognostic biomarker in monitoring of heart allograft rejection, stroke and AMI. This is a crucial result because cfDNA is a potential surrogate for the entire genome and thus a valuable option in cases where tissue quantity is inadequate for mutation testing or in patients who refuse or are unable to undergo EMB. Remarkably, a recent work by Sun et al has demonstrated that another epigenetic biomarker, such as cfDNA fragmentation pattern, could also be informative for $\mathrm{CV}$ diagnosis besides the above-mentioned DNA methylation. ${ }^{114}$

Owing to their reversibility, most attention should be given to circulating epigenetic-sensitive changes, which may provide innovative drug targets. In particular, individual DNA methylation profiles may be useful to identify a personalised dietary interventions consisting of consuming foods containing methyl donors to attenuate cardiac phenotypes. Since epigenetic changes can be mitotically and meiotically inherited across several generations, a liquid biopsy approach could be used to follow this 'transgenerational effect' in families at high risk of CV events to improve primary prevention. ${ }^{7}$ It is well known that a common pitfall of assay development and validation is the comparison to a single current test, including EMB. This is not how medicine should be practised. All of the diagnoses discussed earlier would be approached through a combination of clinical impression/ symptoms, ECG, current laboratory panels and extensive imaging, sometimes supplemented by biopsy. For example, current recommendations for myocarditis management do not even recommend biopsy for most cases given the extensive advances in imaging tools. As such, the focus in clinical trials should be on what the liquid-based assays add, not replace. Another limitation arises from the evidence that liquid diagnoses are largely focused on ischaemic disease, while EMB is almost exclusively indicated for non-ischaemic disease (and largely contraindicated in ischaemic disease). Thus, 'comparisons' should be simply to other biomarkers and parameters, including troponin, brain natriuretic peptide, ECG, echo and cath.

\section{CONCLUSIONS}

Despite many examples of successful applications, there are several barriers to implementation of fluid-based assays into
Take home messages

- Fluid-based assays may provide novel useful non-invasive indicators of cardiovascular diseases.

- NGS platforms performed on body fluid samples are providing a huge amount of big data.

- Potent bioinformatic network-oriented algorithms and artificial intelligence are useful tools to analyse omics data.

- These efforts may provide novel circulating non-invasive biomarkers to realize the goals of $\mathrm{CV}$ personalized therapy.

clinical trials and subsequently into routine CV clinical practice. The different liquid biopsy platforms reviewed here may potentially complement each other for the management of $\mathrm{CV}$ patients. Importantly, we highlight that an integrated approach combining liquid biopsy imaging and networkoriented analyses is the most fruitful way to reach precision medicine and personalised therapy in CV diseases and in oncology, where there is now a major interest despite the fact that solid biopsy is well established and show high accuracy in diagnosis and prognosis.

Sources of Fundings

\section{Handling editor Runjan Chetty.}

Contributors CN: genesis of idea. GB and GM: review of literature, design of tables and figures, initial draft of the manuscript. $\mathrm{CN}$ : critical and final review of the paper.

Funding This work was supported by 'PRIN2017F8ZB89' from Italian Ministry of Research (PI CN). GB is a PhD student of Translational Medicine awarded ESC Congress 2019 Travel Grant and she is supported by Educational Grant from the University of Campania, Naples, Italy.

Disclaimer The funders had no role in study design, data collection and analysis, decision to publish or preparation of the manuscript.

Competing interests None declared.

Patient consent for publication Not required.

Provenance and peer review Not commissioned; externally peer reviewed.

\section{ORCID iDs}

Giuditta Benincasa http://orcid.org/0000-0002-7552-3522

Gelsomina Mansueto http://orcid.org/0000-0002-0544-5100

\section{REFERENCES}

1 Piñero J, Bravo Àlex, Queralt-Rosinach N, et al. DisGeNET: a comprehensive platform integrating information on human disease-associated genes and variants. Nucleic Acids Res 2017;45:D833-D839

2 Leopold JA, Loscalzo J. Emerging role of precision medicine in cardiovascular disease. Circ Res 2018:122:1302-15.

3 Gronda E, Vanoli E, Sacchi S, et al. Risk of heart failure progression in patients with reduced ejection fraction: mechanisms and therapeutic options. Heart Fail Rev $2019 ; 119$.

4 Gronda E, Sacchi S, Benincasa G, et al. Unresolved issues in left ventricular postischemic remodeling and progression to heart failure. J Cardiovasc Med 2019

5 Napoli C, Schiano C, Soricelli A. Increasing evidence of pathogenic role of the mediator (Med) complex in the development of cardiovascular diseases. Biochimie 2019;165:1-8.

6 Schiano C, Costa V, Aprile M, et al. Heart failure: pilot transcriptomic analysis of cardiac tissue by RNA-sequencing. Cardiol J 2017;24:539-53.

7 Napoli C, Benincasa G, Loscalzo J. Epigenetic inheritance underlying pulmonary arterial hypertension. Arterioscler Thromb Vasc Biol 2019;39:653-64

8 Cifuentes A. Advanced food analysis, foodome and foodomics. Electrophoresis 2018;39:1525-6.

9 Leon-Mimila P, Wang J, Huertas-Vazquez A. Relevance of multi-omics studies in cardiovascular diseases. Front Cardiovasc Med 2019;6.

10 Pepin ME, Ha C-M, Crossman DK, et al. Genome-Wide DNA methylation encodes cardiac transcriptional reprogramming in human ischemic heart failure. Lab Invest 2019:99:371-86.

11 Menche J, Sharma A, Kitsak M, et al. Disease networks. uncovering disease-disease relationships through the incomplete interactome. Science 2015;3471257601. 
12 Lee LY-H, Loscalzo J. Network medicine in pathobiology. Am J Pathol 2019;189:1311-26.

13 Cheng F, Lu W, Liu C, et al. A genome-wide positioning systems network algorithm for in silico drug repurposing. Nat Commun 2019;10:3476.

14 Greene JA, Loscalzo J. Putting the Patient Back Together - Social Medicine, Network Medicine, and the Limits of Reductionism. N Engl J Med 2017;377:2493-9.

15 Awan SE, Sohel F, Sanfilippo FM, et al. Machine learning in heart failure: ready for prime time. Curr Opin Cardiol 2018;33:190-5.

16 Butler J, Hamo CE, Udelson JE, et al. Reassessing phase II heart failure clinical trials: consensus recommendations. Circ Heart Fail 2017:10.

17 Ko Y-A, Hayek S, Sandesara P, et al. Cohort profile: the Emory cardiovascular Biobank (EmCAB). BMJ Open 2017;7:e018753.

18 Wu Y, Byrne EM, Zheng Z, et al. Genome-Wide association study of medication-use and associated disease in the UK Biobank. Nat Commun 1891;2019.

19 Coumans FAW, Brisson AR, Buzas El, et al. Methodological guidelines to study extracellular vesicles. Circ Res 2017;120:1632-48.

20 Farinacci M, Krahn T, Dinh W, et al. Circulating endothelial cells as biomarker for cardiovascular diseases. Res Pract Thromb Haemost 2019;3:49-58.

21 Bustamante A, Mancha F, Macher HC, et al. Circulating cell-free DNA is a predictor of short-term neurological outcome in stroke patients treated with intravenous thrombolysis. J Circ Biomark 2016;5.

22 De Vlaminck I, Valantine HA, Snyder TM, et al. Circulating cell-free DNA enables noninvasive diagnosis of heart transplant rejection. Sci Trans/ Med 2014;6:241 ra77.

23 Khan T, Selvakumar D, Trivedi S, et al. The value of endomyocardial biopsy in diagnosis and guiding therapy. Pathology 2017;49:750-6.

24 Ishibashi-Ueda H, Matsuyama T-aki, Ohta-Ogo K, et al. Significance and value of endomyocardial biopsy based on our own experience. Circ J 2017;81:417-26.

25 Buja M, Butany J. Cardiovascular pathology. 4th Edn, 2016.

26 Marcus Fl, Kenna WJ MC, Basso C, et al. Diagnosis of arrhythmogenic right ventricular cardiomyopathy/dysplasia: proposed modification of the task force criteria. Circulation 2010.

27 Corrado D, Fontaine G, Marcus Fl, et al. Arrhythmogenic right ventricular dysplasia/ cardiomyopathy: need for an international registry. Study Group on arrhythmogenic right ventricular dysplasia/cardiomyopathy of the working groups on myocardial and pericardial disease and arrhythmias of the European Society of cardiology and of the scientific Council on cardiomyopathies of the world heart Federation. Circulation 2000;101:101-6.

28 Ponsiglione A, Puglia M, Morisco C, et al. A unique association of arrhythmogenic right ventricular dysplasia and acute myocarditis, as assessed by cardiac MRI: a case report. BMC Cardiovasc Disord 2016;16:230

29 Caforio ALP, Pankuweit S, Arbustini E, et al. Current state of knowledge on aetiology, diagnosis, management, and therapy of myocarditis: a position statement of the European Society of cardiology Working group on myocardial and pericardial diseases. Eur Heart J 2013;34:2636-48.

30 Butler CR, Savu A, Bakal JA, et al. Correlation of cardiovascular magnetic resonance imaging findings and endomyocardial biopsy results in patients undergoing screening for heart transplant rejection. J Heart Lung Transplant 2015;34:643-50

31 Mingo-Santos S, Moñivas-Palomero V, Garcia-Lunar I, et al. Usefulness of Two-Dimensional Strain Parameters to Diagnose Acute Rejection after Heart Transplantation. J Am Soc Echocardiogr 2015;28:1149-56.

32 Antończyk K, Niklewski T, Antończyk R, et al. Speckle-tracking echocardiography for monitoring acute rejection in transplanted heart. Transplant Proc 2018;50:2090-4.

33 Tebbe U, Bramlage K, John F, et al. Endomyocardial biopsy in patients with cardiomyopathy of unknown origin: does specialized center experience apply to a tertiary care hospital? BMC Res Notes 2016;9:459.

34 Majumdar M, Martin J. Detection by direct next generation sequencing analysis of emerging enterovirus D68 and C109 strains in an environmental sample from Scotland. Front Microbiol 1956;2018.

35 Porzionato A, Macchi V, Stecco C. The body donation program of the University of Padua: organizing an anatomical biobank for medical education. In: Caenazzo L, ed. New insights on biobanks. Padova, Italy: CLEUP, 2013: 155-71.

36 Perakis S, Speicher MR. Emerging concepts in liquid biopsies. BMC Med 2017;15:75

37 Sabia C, Picascia A, Grimaldi V, et al. The epigenetic promise to improve prognosis of heart failure and heart transplantation. Transplant Rev 2017;31:249-56

38 Maron BJ, Maron MS, Maron BA, et al. Moving Beyond the Sarcomere to Explain Heterogeneity in Hypertrophic Cardiomyopathy: JACC Review Topic of the Week. J Am Coll Cardiol 2019;73:1978-86.

39 Jain KK. Personalized management of cardiovascular disorders. Med Princ Pract 2017:26:399-414.

40 Schiano C, Rienzo M, Casamassimi A, et al. Splicing regulators in endothelial cell differentiation. J Cardiovasc Med 2017:18:742-9.

41 Liguori A, Fiorito C, Balestrieri ML, et al. Functional impairment of hematopoietic progenitor cells in patients with coronary heart disease. Eur J Haematol 2008;80:258-64.

42 Li X, Qiu J, Pan M, et al. Correlation between congenital heart disease complicated with pulmonary artery hypertension and circulating endothelial cells as well as endothelin-1. Int J Clin Exp Pathol 2015;8:10743-51.
43 Watt J, Kennedy S, Ahmed N, et al. The relationship between oxidised LDL, endothelial progenitor cells and coronary endothelial function in patients with CHD. Open Heart 2016;3:e000342.

44 Jahr $\mathrm{S}$, Hentze $\mathrm{H}$, Englisch $\mathrm{S}$, et al. Dna fragments in the blood plasma of cance patients: quantitations and evidence for their origin from apoptotic and necrotic cells. Cancer Res 2001:61:1659-65.

45 Sun K, Jiang P, Chan KCA, et al. Plasma DNA tissue mapping by genome-wide methylation sequencing for noninvasive prenatal, cancer, and transplantation assessments. Proc Natl Acad Sci U S A 2015;112:E5503-E5512.

46 Lam WKJ, Gai W, Sun K, et al. Dna of erythroid origin is present in human plasma and informs the types of anemia. Clin Chem 2017;63:1614-23.

47 Xie J, Yang J, Hu P. Correlations of circulating cell-free DNA with clinical manifestations in acute myocardial infarction. Am J Med Sci 2018:356:121-9.

48 Zhou M-Y, Yang J-M, Xiong X-D. The emerging landscape of circular RNA in cardiovascular diseases. J Mol Cell Cardiol 2018;122:134-9.

49 Holdt LM, Stahringer A, Sass K, et al. Circular non-coding RNA ANRIL modulates ribosomal RNA maturation and atherosclerosis in humans. Nat Commun 2016:7:12429.

50 Zhao Z, Li X, Gao C, et al. Peripheral blood circular RNA hsa_circ_0124644 can be used as a diagnostic biomarker of coronary artery disease. Sci Rep 2017;7:39918.

51 Salgado-Somoza A, Zhang L, Vausort M, et al. The circular RNA MICRA for risk stratification after myocardial infarction. Int J Cardiol Heart Vasc 2017;17:33-6.

52 Bao X, Zheng S, Mao S, et al. A potential risk factor of essential hypertension in case-control study: circular RNA hsa_circ_0037911. Biochem Biophys Res Commun 2018;498:789-94

53 Ayupe AC, Reis EM. Evaluating the stability of mRNAs and noncoding RNAs. Methods Mol Biol 2017;1468:139-53.

54 Zemmour H, Planer D, Magenheim J, et al. Non-Invasive detection of human cardiomyocyte death using methylation patterns of circulating DNA. Nat Commun 2018;9:1443.

55 de Nigris F, Cacciatore F, Mancini FP, et al. Epigenetic hallmarks of fetal early atherosclerotic lesions in humans. JAMA Cardiol 2018:3:1184-91.

56 Napoli C, Crudele V, Soricelli A, et al. Primary prevention of atherosclerosis: a clinical challenge for the reversal of epigenetic mechanisms? Circulation 2012;125:2363-73.

57 Zhong J, Agha G, Baccarelli AA. The role of DNA methylation in cardiovascular risk and disease: methodological aspects, study design, and data analysis for epidemiological studies. Circ Res 2016;118:119-31.

58 Schiano C, Vietri MT, Grimaldi V, et al. Epigenetic-related therapeutic challenges in cardiovascular disease. Trends Pharmaco/ Sci 2015;36:226-35.

59 Di Salvo TG, Haldar SM. Epigenetic mechanisms in heart failure pathogenesis. Circ Heart Fail 2014:7:850-63.

60 Carthew RW, Sontheimer EJ. Origins and mechanisms of miRNAs and siRNAs. Cell 2009:136:642-55.

61 Zhou S-S, Jin J-P, Wang J-Q, et al. miRNAS in cardiovascular diseases: potential biomarkers, therapeutic targets and challenges. Acta Pharmacol Sin 2018;39:1073-84.

62 Wang W, Li Z, Zheng Y, et al. Circulating microRNA-92a level predicts acute coronary syndrome in diabetic patients with coronary heart disease. Lipids Health Dis 2019;18:22.

63 Marfella R, Di Filippo C, Potenza N, et al. Circulating microRNA changes in heart failure patients treated with cardiac resynchronization therapy: responders vs. nonresponders. Eur J Heart Fail 2013:15:1277-88.

64 Ovchinnikova ES, Schmitter D, Vegter EL, et al. Signature of circulating microRNAs in patients with acute heart failure. Eur J Heart Fail 2016:18:414-23.

65 Masson S, Batkai S, Beermann J, et al. Circulating microRNA-132 levels improve risk prediction for heart failure hospitalization in patients with chronic heart failure. Eur $J$ Heart Fail 2018;20:78-85.

66 Bayés-Genis A, Lanfear DE, de Ronde MWJ, et al. Prognostic value of circulating microRNAs on heart failure-related morbidity and mortality in two large diverse cohorts of general heart failure patients. Eur J Heart Fail 2018:20:67-75.

67 Benincasa G, Costa D, Infante T, et al. Interplay between genetics and epigenetics in modulating the risk of venous thromboembolism: a new challenge for personalized therapy. Thromb Res 2019;177:145-53.

68 Gao H, Zhang N, Lu F, et al. Circulating histones for predicting prognosis after cardiac surgery: a prospective study. Interact Cardiovasc Thorac Surg 2016;23:681-7

69 Qi Y, Uchida T, Yamamoto M, et al. Perioperative elevation in cell-free DNA levels in patients undergoing cardiac surgery: possible contribution of neutrophil extracellular traps to perioperative renal dysfunction. Anesthesiol Res Pract 2016:2016:1-11.

70 Fritz KS. Chemical acetylation and deacetylation. Methods Mol Biol 2013:1077:191-201.

71 Schiattarella GG, Sannino A, Toscano E, et al. Cardiovascular effects of histone deacetylase inhibitors epigenetic therapies: systematic review of 62 studies and new hypotheses for future research. Int J Cardiol 2016;219:396-403.

72 Mega JL, Stitziel NO, Smith JG, et al. Genetic risk, coronary heart disease events, and the clinical benefit of statin therapy: an analysis of primary and secondary prevention trials. The Lancet 2015;385:2264-71. 
73 Sen-Banerjee S, Mir S, Lin Z, et al. Kruppel-Like factor 2 as a novel mediator of statin effects in endothelial cells. Circulation 2005;112:720-6.

74 Jukema JW, Cannon CP, de Craen AJM, et al. The controversies of statin therapy: weighing the evidence. J Am Coll Cardiol 2012;60:875-81.

75 Lawson C, Vicencio JM, Yellon DM, et al. Microvesicles and exosomes: new players in metabolic and cardiovascular disease. J Endocrinol 2016;228:R57-R71.

76 Chistiakov D, Orekhov A, Bobryshev Y. Cardiac extracellular vesicles in normal and infarcted heart. Int J Mol Sci 2016;17:63.

77 Jansen F, Nickenig G, Werner N. Extracellular vesicles in cardiovascular disease: potential applications in diagnosis, prognosis, and epidemiology. Circ Res 2017;120:1649-57.

78 Davidson SM, Yellon DM. Exosomes and cardioprotection - A critical analysis. Mol Aspects Med 2018;60:104-14.

79 Jansen F, Yang X, Proebsting S, et al. Microrna expression in circulating microvesicles predicts cardiovascular events in patients with coronary artery disease. J Am Heart Assoc 2014;3:e001249.

80 Yang Y, Li Y, Chen X, et al. Exosomal transfer of miR-30a between cardiomyocytes regulates autophagy after hypoxia. J Mol Med 2016;94:711-24.

81 Matsumoto S, Sakata Y, Suna S, et al. Circulating p53-responsive microRNAs are predictive indicators of heart failure after acute myocardial infarction. Circ Res 2013;113:322-6.

82 Dougherty JA, Mergaye M, Kumar N, et al. Potential role of exosomes in mending a broken heart: nanoshuttles propelling future clinical therapeutics forward. Stem Cells Int 2017:2017:1-14.

83 Bellin G, Gardin C, Ferroni L, et al. Exosome in cardiovascular diseases: a complex world full of hope. Cells 2019:8.

84 Jung J-H, Fu X, Yang PC. Exosomes generated from iPSC-derivatives: new direction for stem cell therapy in human heart diseases. Circ Res 2017;120:407-17.

85 McGarrah RW, Crown SB, Zhang G-F, et al. Cardiovascular metabolomics. Circ Res 2018;122:1238-58.

86 Wishart DS, Feunang YD, Marcu A, et al. HMDB 4.0: the human metabolome database for 2018. Nucleic Acids Res 2018:46:D608-D617.

87 Würtz P, Havulinna AS, Soininen P, et al. Metabolite profiling and cardiovascular event risk: a prospective study of 3 population-based cohorts. Circulation 2015:131:774-85.

88 Ganna A, Salihovic S, Sundström J, et al. Large-Scale metabolomic profiling identifies novel biomarkers for incident coronary heart disease. PLoS Genet 2014; 10:e1004801

89 Stenemo M, Ganna A, Salihovic S, et al. The metabolites urobilin and sphingomyelin (30:1) are associated with incident heart failure in the general population. ESC Heart Fail 2019;6:764-73.

90 Gohel V, Jones JA, Wehler CJ. Salivary biomarkers and cardiovascular disease: a systematic review. Clin Chem Lab Med 2018;56:1432-42.

91 Rahim MAA, Rahim ZHA, Ahmad WAW, et al. Can saliva proteins be used to predict the onset of acute myocardial infarction among high-risk patients? Int J Med Sci 2015:12:329-35

92 Foo JYY, Wan Y, Kostner K, et al. Nt-Probnp levels in saliva and its clinical relevance to heart failure. PLoS One 2012:7:e48452.

93 Miller CS, Foley JD, Floriano PN, et al. Utility of salivary biomarkers for demonstrating acute myocardial infarction. J Dent Res 2014;93(7 Suppl):72S-9.

94 Labat C, Temmar M, Nagy E, et al. Inflammatory mediators in saliva associated with arterial stiffness and subclinical atherosclerosis. J Hypertens 2013:31:2251-8.

95 Bazzell BG, Rainey WE, Auchus RJ, et al. Human urinary mRNA as a biomarker of cardiovascular disease. Circ Genom Precis Med 2018;11:e002213.

96 Napoli C, Zullo A, Picascia A, et al. Recent advances in proteomic technologies applied to cardiovascular disease. J Cell Biochem 2013;114:7-20.

97 Röthlisberger S, Pedroza-Diaz J. Urine protein biomarkers for detection of cardiovascular disease and their use for the clinic. Expert Rev Proteomics 2017;14:1091-103.
98 Ristevski B, Chen M. Big data analytics in medicine and healthcare. J Integr Bioinform 2018;15. doi:10.1515/jib-2017-0030. [Epub ahead of print: 10 May 2018].

99 Morris MA, Saboury B, Burkett B, et al. Reinventing radiology: big data and the future of medical imaging. J Thorac Imaging 2018;33:4-16.

100 Maheshwari A, Norby FL, Roetker NS, et al. Refining prediction of atria fibrillation-related stroke using the P(2)-CHA(2)DS(2)-VASc Score. Circulation 2019:139:180-91.

101 Kormeier B, Hippe K, Töpel T, et al. CardioVINEdb: a data Warehouse approach for integration of life science data in cardiovascular diseases. J Integr Bioinform 2010;7:142.

102 Hsu PD, Lander ES, Zhang F. Development and applications of CRISPR-Cas9 for genome engineering. Cell 2014;157:1262-78.

103 Fang J, Cai C, Chai Y, et al. Quantitative and systems pharmacology 4. networkbased analysis of drug pleiotropy on coronary artery disease. Eur J Med Chem 2019;161:192-204.

104 Infante T, Forte E, Punzo B, et al. Correlation of circulating miR-765, miR-93-5p, and miR-433-3p to obstructive coronary heart disease evaluated by cardiac computed tomography. Am J Cardiol 2019:124:176-82.

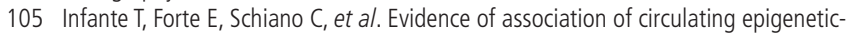
sensitive biomarkers with suspected coronary heart disease evaluated by cardiac computed tomography. PLoS One 2019;14:e0210909.

106 Azuaje FJ, Rodius S, Zhang L, et al. Information encoded in a network of inflammation proteins predicts clinical outcome after myocardial infarction. BMC Med Genomics 2011;4:59.

107 Azuaje F, Zhang L, Jeanty C, et al. Analysis of a gene co-expression network establishes robust association between COL5A2 and ischemic heart disease. BMC Med Genomics 2013:6:13.

108 Hartmann K, Seweryn M, Handelman SK, et al. Non-Linear interactions between candidate genes of myocardial infarction revealed in mRNA expression profiles. BMC Genomics 2016;17:738.

109 Jin G, Zhou X, Wang H, et al. The knowledge-integrated network biomarkers discovery for major adverse cardiac events. J Proteome Res 2008;7:4013-21.

110 Wang J, Peng $X$, Peng W, et al. Dynamic protein interaction network construction and applications. Proteomics 2014:14:338-52.

111 Liu R, Li M, Liu Z-P, et al. Identifying critical transitions and their leading biomolecular networks in complex diseases. Sci Rep 2012;2:813.

112 Zeller T, Hughes M, Tuovinen T, et al. BiomarCaRE: rationale and design of the European BiomarCaRE project including 300,000 participants from 13 European countries. Eur J Epidemiol 2014;29:777-90.

113 Deng MC, Eisen HJ, Mehra MR, et al. Noninvasive discrimination of rejection in cardiac allograft recipients using gene expression profiling. Am J Transplant 2006;6:150-60.

114 Sun K, Jiang P, Cheng SH, et al. Orientation-aware plasma cell-free DNA fragmentation analysis in open chromatin regions informs tissue of origin. Genome Res 2019;29:418-27.

115 Agbor-Enoh S, Tunc I, De Vlaminck I, et al. Applying rigor and reproducibility standards to assay donor-derived cell-free DNA as a non-invasive method for detection of acute rejection and graft injury after heart transplantation. J Heart Lung Transplant 2017:36:1004-12.

116 Devaux Y, Dankiewicz J, Salgado-Somoza A, et al. Association of circulating microRNA-124-3p levels with outcomes after out-of-hospital cardiac arrest: a substudy of a randomized clinical trial. JAMA Cardio/ 2016;1:305-13.

117 Chao Ting-Hsing, Chen I-Chih, Li Yi-Heng, et al. Plasma levels of proprotein convertase subtilisin/kexin type 9 are elevated in patients with peripheral artery disease and associated with metabolic disorders and dysfunction in circulating progenitor cells. J Am Heart Assoc 2016;5.

118 Ç B, Durdu S, Dalva K, et al. Effects of preoperative short term use of atorvastatin on endothelial progenitor cells after coronary surgery: a randomized, controlled trial. Stem Cell Rev 2012;8:963-71. 